\title{
A Wide Tuning Range Rf Cavity with External Ferrite Biasing*
}

\author{
$X$. Pei* \\ Brookhaven National Laboratory, Upton, NY 11973, USA \\ S. Anderson, D. Jenner, D. McCammon, T. Sloan \\ Indiana University Cyclotron Facility, Bloomington, IN 47405, US

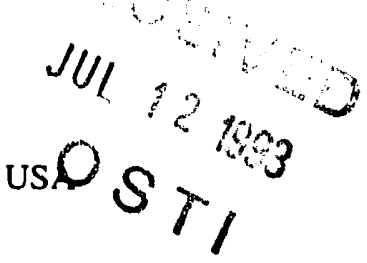

Abstract

A wide tuning range if cavity with external quadrupole ferrite biasing was successfully built and has been in operation in the Indiana University Cooler Synchrotron Ring. The new biasing scheme was proposed by S. Papureanu and allows a large number of turns on the biasing coils. Large ampere-turns achieving strong biasing $B$ field can therefore be achieved by a power source of much smaller current rating. The external biasing also makes the if cavity design straightforward, eliminating biasing structure related parasitic effects, such as hard to predict high order modes.

\section{INTRODUCTION}

The electron-cooled Indiana University Cooler Synchrotron has a circumference of $86.8 \mathrm{~m}$ and a maximum rigidity of $3.6 \mathrm{~T}-\mathrm{m}$. It was designed with long straight sections free of beam optical elements to suit the internal target nuclear physics experiments. When its longitudinal emittance is reduced by electron cooling, the beam forms a tight bunch in the center of an if bucket. Operation of the if system at the fundamental beam revolution frequency makes the beam of the entire ring congregate as a macro particle. This allows certain operations of the ring that are inevitably lossy, such as transverse kickers for multi-turn stacking injection, to avoid the beam azimuth position to minimize beam loss. An if system capable of $h=1$ operation is therefore introduced in addition to the existing if system. It was alsc hoped that the new rf system has a wider tunable frequency swing to accommodate acceleration of heavier-than-proton particles. The frequency swing of the existing if system is limited to an approximate 2:1 ratio with a bulky power supply rated at 4000 Amperes.

\section{CAVITY DESIGN}

Based on the revolution frequency of $45 \mathrm{MeV}$ protons in the $86.8 \mathrm{~m}$ ring, the lower frequency limit of the cavity was chosen at $0.5 \mathrm{MHz}$. Fast tuning of the cavity is accomplished by utilizing the non-linear permeability characteristics of ferrite materials. At a higher magnetic field, the permeability of ferrites decreases and eventually approaches zero due to field saturation. Thus a biasing DC field can be used to tune the cavity. Although the art of ferrite biasing has been tried for

\footnotetext{
* Work supported in part by NSF PHY9015957.

Work performed under the auspices of the U.S. Department of Energy.
}

decades, a scheme proposed by S. Papureanu and first adopted at Max Planck Institute appeared to suit our application especially well. Because of the origin, the cavity was named the MPI cavity. Fig.1 shows the principle of this biasing scheme.

The of cavity is loaded with ferrite rings for tuning and for shortening the length required for a quarter wave coaxial resonant structure at $0.5 \mathrm{MHz}$. The outer conductor of the coaxial cavity is made of copper which is non-ferromagnetic. The entire cavity then is placed within a quadrupole magnet cut to fit the cavity. 40 turns of windings are put on each tip of the quadrupole. The biasing magnetic field path is shown in Fig.1. The center of the ferrite ring where the beam pipe is located is well protected from the biasing field due to a), the cancellation of field due to quadrupole field symmetry and b), the shielding and shunting of high permeability ferrite rings. Because the biasing structure is totally outside the if cavity, it is not seen by the if field. The if design of the cavity is therefore clean and does not suffer from the parasitic effects brought in by the biasing structure. In addition, the entirely external biasing structure has sufficient space to allow a large number of bias winding turns. This is crucial to the use of a much smaller power supply rated at 20 Amperes as the biasing magnetic field strength depends on the total ampere turns of the bias windings.

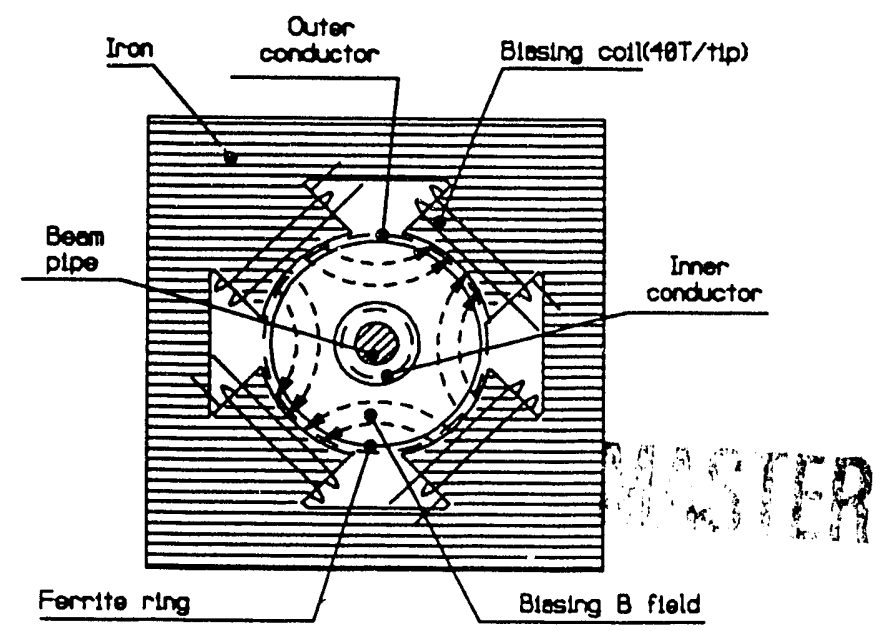

Fig.l Principle of external quadrupole magnet ferrite biasing.

An additional variable capacitor ( $4100 \mathrm{pf}$ maximum capacitance) is added across the gap to reach a resonant frequency of $0.5 \mathrm{MHz}$ and to make the cavity capable of covering an even wider tuning range. The heavy capacitative

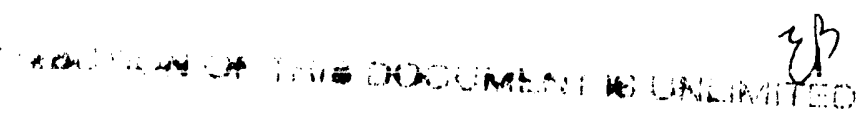


loading of the cavity also makes the cavity store predominantly magnetic energy (short transmission line approximation). Therefore it has the additional advantage of making the driving port of the cavity, which consists of a magnetic loop connected to the center conductor of the cavity, have a relatively constant impedance to match the $50 \mathrm{Ohm}$ feeding transmission lines. Cooling of the cavity is via forced air. The ferrite rings have spaces between them. The center conductor of the cavity is perforated. And the oute $i$ conductor of the cavity is made of copper straps with spaces in between rather than solid copper. A fan forces air through all these air passages and keeps the ferrite and the cavity cool. Fig. 2 is a cutaway illustration of the cavity design. The diameter of the cavity is about 0.55 meters and the length 0.6 meters. A total of 10 Philips accelerator ferrite rings, material: $8 \mathrm{C} 12$; dimensions: $\$ 498 \times \$ 270 \times 25$ $\mathrm{mm}$, were used.

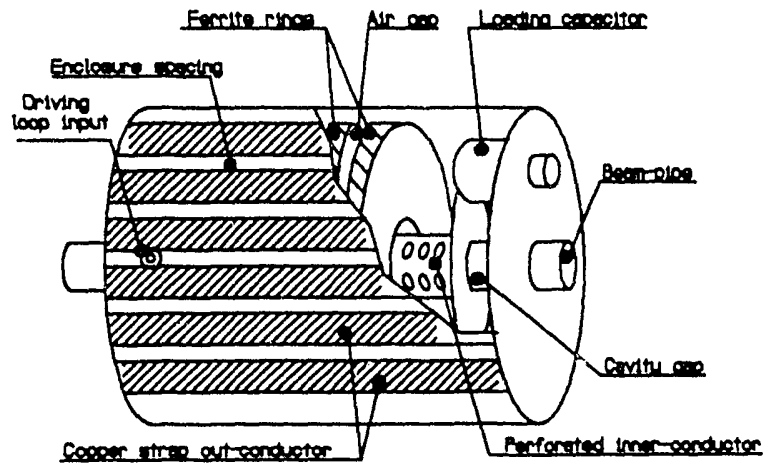

Fig.2. Cutaway illustration of the MPl cavity showing its internal structure.

The bottom outer conductor was partially removed to allow support of the cavity. The loading capacitor across the cavity gap is placed above the beam pipe. The driving loop is a simple side-insertion type. All these severely break the symmetry of the cavity so the small if leakage inherent with coaxial structures no longer holds true. The cavity has to be shielded against if radiation and aluminum lids were placed on both the front and back sides of the biasing quadrupole as shown in Fig.3. The quadrupole itself serves as if shield in the center.

\section{PERFORMANCE MEASUREMENTS}

In the vicinity of resonance, the cavity can be modeled with a lossy shunt $L-C-R$ circuit. The $L C$ part determines the resonant frequency. Accurate to the first order in our case, the $L$ value is variable by the ferrite biasing field and the $C$ value is determined by the mechanical tuning of the variable capacitor. For our application, we are also very concerned with the field strength developed across the gap at a given driving power.
Because power dissipation only occurs on the resistor, we call the shunt resistor "gap resistance" and is defined as $R_{g}$ as follows:

$$
R_{8}=\frac{V_{g}^{2}}{2 P}
$$

where $V_{g}$ is the total voltage developed across the gap of the cavity and $P$ the total power absorbed by the cavity. It is obvious that for higher $R_{\mathrm{g}}$ 's, less power is needed to drive the cavity to desired voltage.

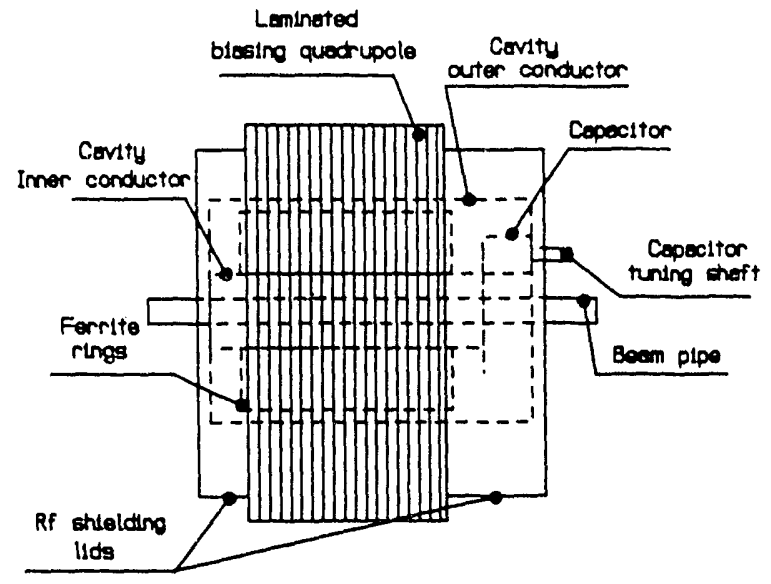

Fig.3 Sideview of the cavity with if shielding lids.

Fig. 4 through Fig.7 are measured frequency versus bias supply current plots and frequency versus gap resistance plots at four different loading capacitor settings. Note that the effective ampere-turn is 40 times of the bias supply current as the quadrupole magnet has 40 turns of windings on each of its tip.

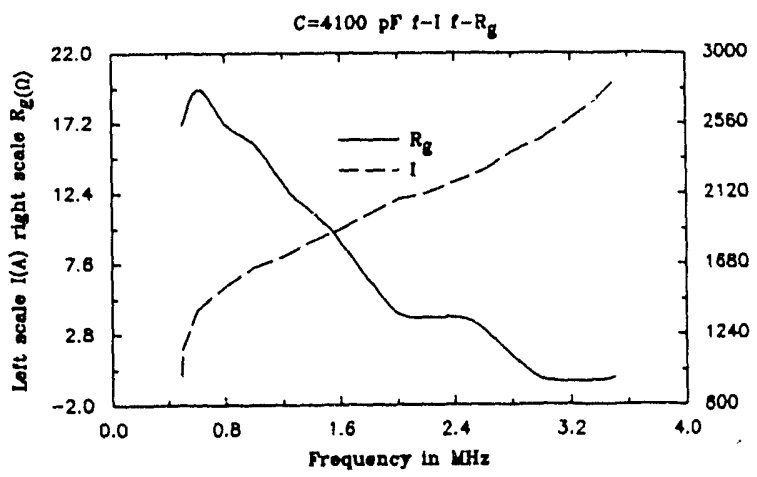

Fig.4 Frequency versus bias supply current and frequency versus gap resistance at $C=4100 \mathrm{pf}$. 


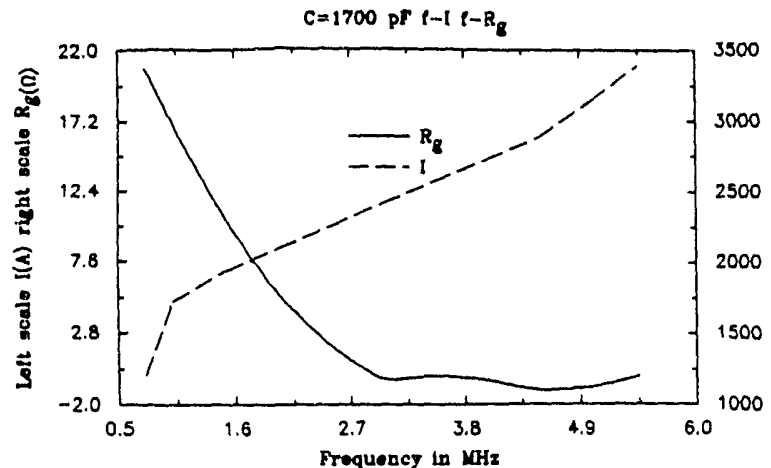

Fig.5 Frequency versus bias supply current and frequency versus gap resistance at $C=1700 \mathrm{pf}$.

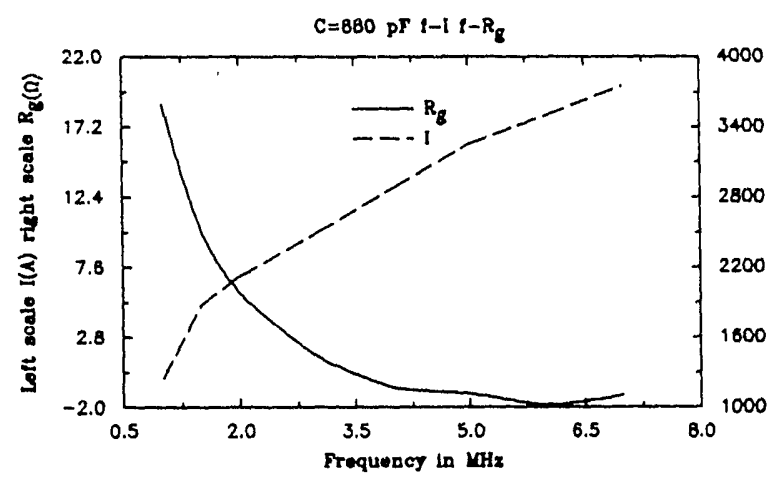

Fig. 6 Frequency versus bias supply current and frequency versus gap resistance at $C=880 \mathrm{pf}$.

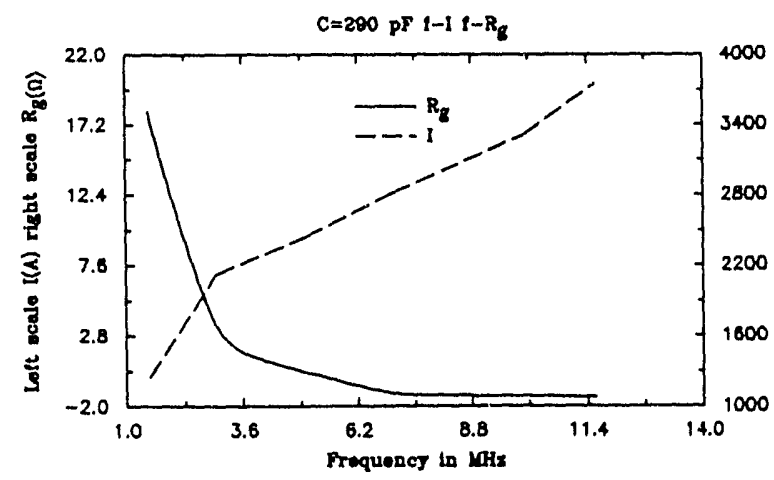

Fig.7 Frequency versus bias supply current and frequency versus gap resistance at $C=290$ pf.

\section{ACKNOWLEDGEMENTS}

This work was supported by the U.S. National Science Foundation under grant NSF PHY9015957 and was partially funded by Indiana University and the State of Indiana. The authors wish to thank R. E. Pollock for suggesting the MPI cavity design and calculating key specifications. This work was made possible by the concerted efforts of all the staff of the Indiana University Cyclotron Facility.

\section{REFERENCES}

[1] Pei, X., "Cooled Rf Stacking Injection in the IUCF Cooler," Ph.D. Dissertation, Indiana University, 1991.

[2] Pei, X. et. al., 'Low Frequency Rf System for the Indiana University Cooler Ring", 13th International Conference on Cyclotrons, Vancouver, Canada, 1992.

[3] Pollock, R. E., "Cooler Design Notes," IUCF, 1988. 

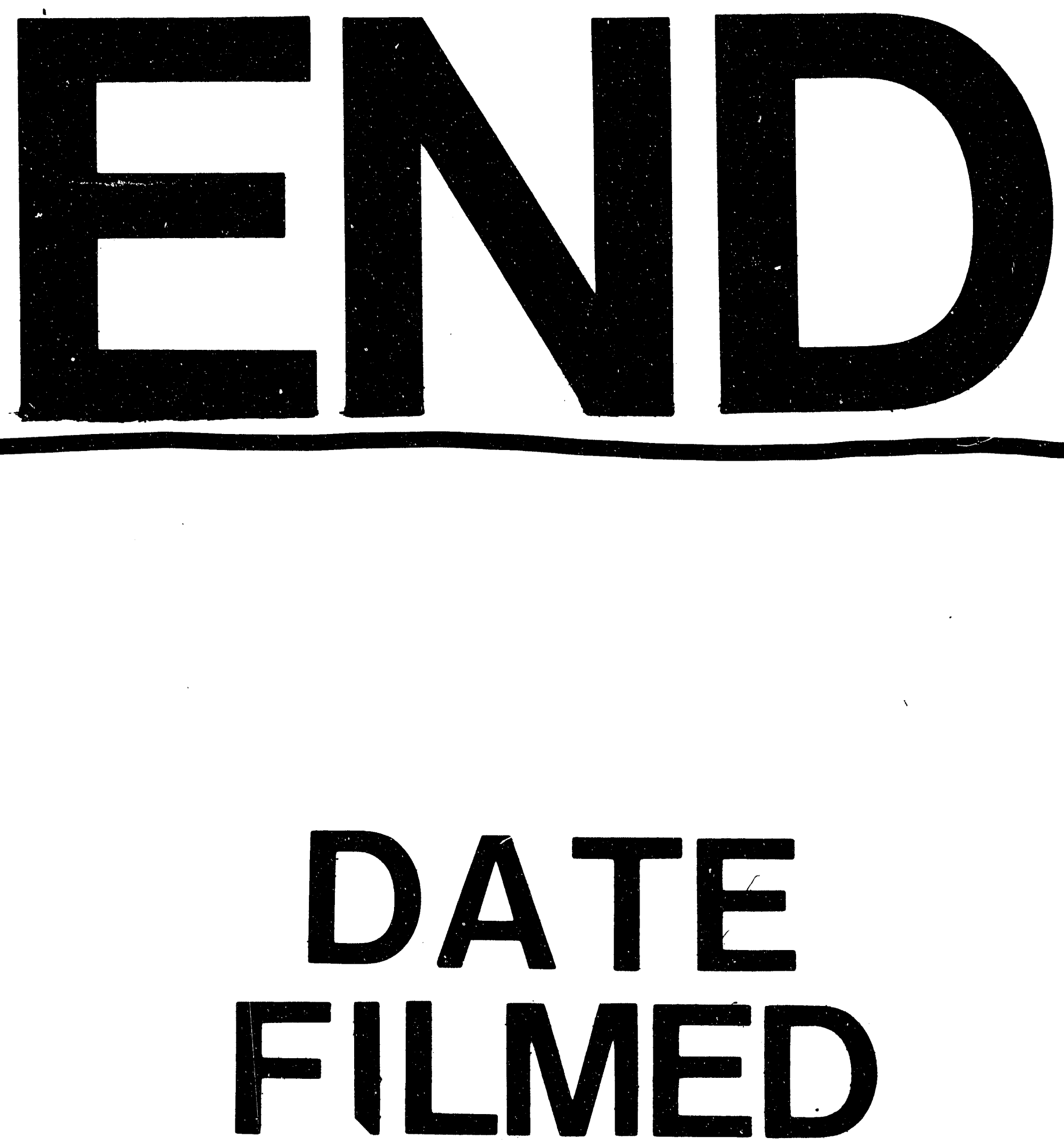

9
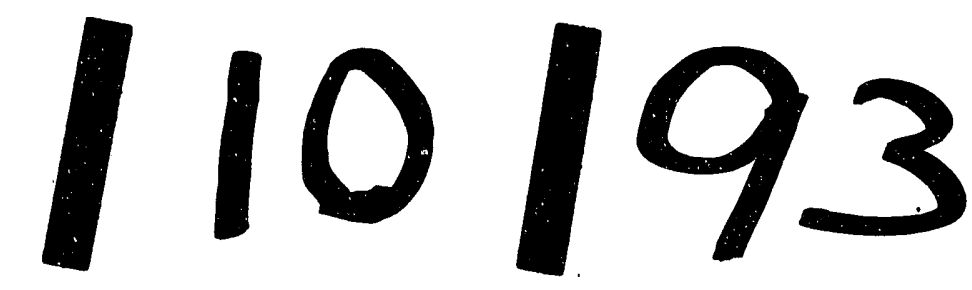
